\title{
12-lead ECG Arrythmia Classification Using Convolutional Neural Network for Mutually Non-Exclusive Classes
}

\author{
Mateusz Soliński ${ }^{1}$, Michał Łepek ${ }^{1}$, Antonina Pater ${ }^{1}$, Katarzyna Muter $^{2}$, Przemysław Wiszniewski ${ }^{3}$ \\ Dorota Kokosińska ${ }^{1}$, Judyta Salamon ${ }^{1}$, Zuzanna Puzio ${ }^{1}$ \\ ${ }^{1}$ Faculty of Physics, Warsaw University of Technology \\ ${ }^{2}$ Faculty of Electronics and Information Technology, Warsaw University of Technology \\ ${ }^{3}$ Faculty of Electrical Engineering, Warsaw University of Technology
}

\begin{abstract}
The growing demand for diagnosing of cardiovascular diseases leads to the development of new solutions for automatic classification of recorded ECG signals. Creating a robust and fast algorithm for automatic classification of ECG signal is crucial to improve the quality of healthcare, especially in countries where a lack of experienced specialists is an issue or the healthcare system is overloaded. The aim of the PhysioNet/Computing in Cardiology Challenge 2020 is to create an algorithm for classification of 12-lead ECGs based on ECG signals from multiple databases across the world. The shared training set consisted of 43,101 ECG recordings lasting from 5 to 1800 seconds. We (BioS Team) proposed the machine learning algorithm based on convolutional neural networks. The ECG signals were pre-processed using moving median filters to remove high-frequency noise and baseline wandering. We developed simply convolutional neural network consisting of four main convolutional blocks and one fully connected layer. We achieved a challenge validation score of 0.349 , and full test score of 0.279 , placing us 14 out of 41 in the official ranking.
\end{abstract}

\section{Introduction}

Cardiovascular diseases (CVDs) are the leading cause of death worldwide, killing about 17.9 million lives each year (WHO, 2016). A Standard tool for diagnosing of many CVDs is 12-lead electrocardiogram (ECG). In a traditional way, 12-ECG records are interpreted by cardiologists manually based on diagnosis criteria and personal experience. However, manual interpretation of the recordings is time-consuming and requires high degree of skills. The growing demand for diagnostic testing is leading to the development of new solutions for automatic classification of recorded signals.

Since late 1950s, the performance of automatic algorithm for ECG interpretation were grown with computational capacity of the computers and developing new methods of analysis, preprocessing and classification [1]. Many algorithms are based on three processes: feature extraction, feature selection, and classification [2]. The alternative way is to use the machine learning methods, e.g.: convolutional neural networks (CNN), which do not need prior defined features and take to the input the raw (or preprocessed) signal [3].

Development of automatic ECG classifier was the aim of PhysioNet/Computing in Cardiology Challenge 2020 [4]. Participants had to deal with the problem of creating an algorithm that recognizes 27 classes using multiple databases from across the world.

In this article we present an algorithm for multilabel classification using machine learning method based on the $(\mathrm{CNN})$. We described the details of the algorithm, necessary for reproducing, and showed its limitations and suggestions for its improvement.

\section{Data}

The data, shared for the training, came from 4 sources: CPSC - database from the China Physiological Signal Challenge 2018 (CPSC2018) [5], INCART public dataset from the St. Petersburg Institute of Cardio-logical Technics (Russia) [6,7], PTB and PTB-XL - Physikalisch Technische Bundesanstalt (PTB) Database, Brunswick, German [8,9] and G12EC Georgia 12-leadECG Challenge (G12EC) Database, Emory University, Atlanta, Georgia, USA. Total number of recordings from these databases was 43,101. Each recording contains, apart from ECG signals, information about age, sex and diagnoses. From total number of 111 diagnoses, only 27 were considered by the scoring system (all classes are listed in [4]). The recordings differ in regard to the duration $(5-1800 \mathrm{~s}$, mean: $15.8 \pm 75.9 \mathrm{~s})$ and sample frequency $(257-1000 \mathrm{~Hz})$. 


\section{Methods}

\subsection{Preprocessing}

Initially all signals are resampled to $100 \mathrm{~Hz}$ and cut at $10 \mathrm{~s}$ (if any signal was shorter it is copied enough times to fill in 10 seconds). Next, the processing is done in two steps:

- each channel the signals are smoothened using moving median filter with window of 5 samples.

- $\quad$ signals are detrended by subtracting a moving mean with window size of 50 samples.

Next, input to the CNN network as 2D matrix was prepared. The subsequent ECG leads were placed in the rows of this matrix. Graphical representation of the input matrix was presented in Figure 1.
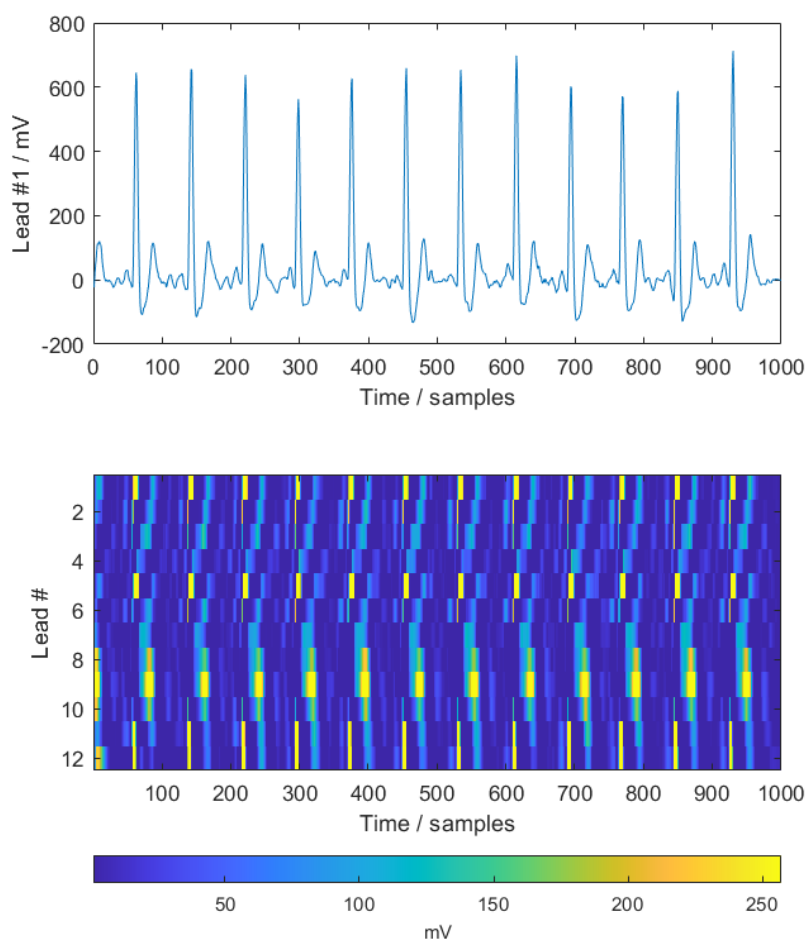

Figure 1. Example of the 10-secods ECG signal from the Lead 1 (upper) and single data input to the CNN network consisted of the 12 ECG leads merged in one 2D matrix.

\subsection{CNN algorithm}

The architecture of our model is shown in Figure 2. We used two-dimensional convolutional neural network for pre-processed 12-lead ECG signal. The architecture consisted of four convolutional blocks followed by a fully connected layer. In each convolutional block there was double two-dimensional convolution operations followed by ReLU activation functions, max-pooling layer and dropout layer (10\% of dropout), except for the last block where max-pooling was not used because maximum level of compression of the data was achieved. In all four blocks 8, 16, 32, 64 convolution filters were used, respectively. The size of convolution kernels in all convolutional layers was set to $3 \times 3$. We used the "same" size of the zero padding in convolutional layers so that the layer output has the same size as the input. The maxpooling with size $2 \times 2$ and stride of 2 was used to reduce the dimension of each convolutional block output.

The number of neurons in fully connected layer was equal to the number of classes (27). The sigmoid activation (instead of softmax) was applied to all output values from last fully connected layer. The signal was classified as the categories whose sigmoid output value exceeded 0.35 .

We chose L2 regularization as a regularization technique in addition to dropout. We set $\mathrm{L} 2$ regularization factor to 0.06 .

We trained out network for 30 epochs with mini-batch size equal 1024. The training data set was randomly split into $80 \%$ training and $20 \%$ validation. Classification was carried out using categorical-cross-entropy loss function and ADAM optimizer with the following parameters: learning rate $=0.001$, epsilon $=0.001$, beta $1=0.9$, beta $2=0.9$.

Network architecture and hyperparameters were finetuned manually through a limited trial and error process. We have not tried to test many other potentially good models, neither with similar nor very different architecture. All hyperparameter values for the proposed network are summarized in Table 1.

The model was developed and implemented in MATLAB 2020a environment. To train our network we used so-called Custom Training Loop to implement mutually non-exclusive classification (more than one class can occur in a single ECG recording, e.g., sinus rhythm and single premature ventricular contraction, PVC). 


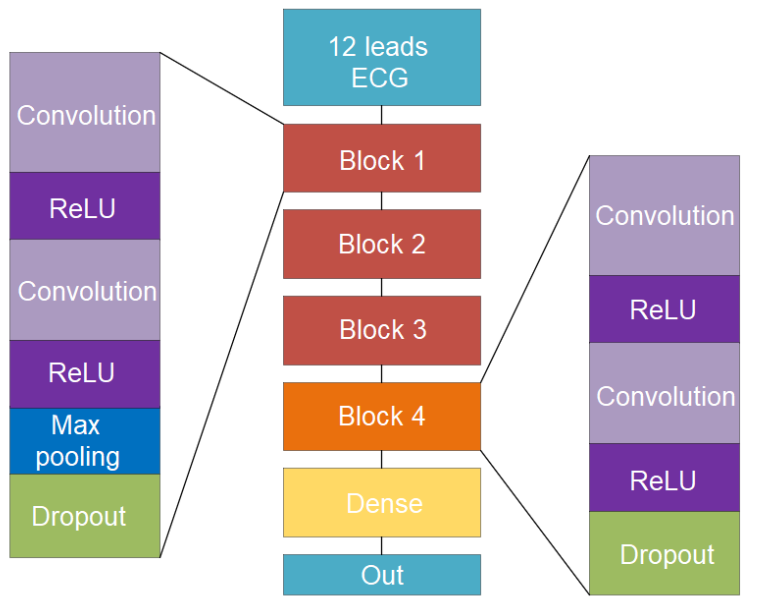

Figure 2. Architecture of the classification model. The model consisted of 4 blocks. Each block contains two convolution and ReLU layers, one max pooling and one dropout (10\% of dropout) layer. The exception is the $4^{\text {th }}$ block where the max pooling layer is not present.

\section{Results}

Our approach achieved a challenge validation score of 0.349 , and full test score of 0.279 , placing us 14 out of 41 in the official ranking of the Challenge.

\section{Discussion}

The method used in this work can be regarded as a relatively simply convolutional neural network with a superficial preprocessing as the network consisted of 4 main convolutional blocks and 1 fully connected layer only. Similar networks has been used for a wide variety of problems before and, here, for ECG arrythmias, it has shown moderate performance of 0.349 (the challenge metric) on the hidden test set. The challenge score obtained from this set is smaller than the score calculated for the data shared for training. It may suggest that the model is overfitting and additional steps for increasing generalization abilities of the classification model.

Despite its simple architecture and limited overall performance, some significant observations can be made. The performance of classification varied for different types of arrythmias. The best performance was obtained for AF, LBBB, PR, RBBB, SNR, and STach. On the other hand, the classifier totally failed to detect some other arrythmias such as CRBBB, LAnFB, LQRSV, NSIVCB, PVC, RAD, SA, SVPB, TInv, and VPB. There are few reasons for that. Some of the weakest-performing classes were significantly underrepresented in the dataset.
Table 1. Numbers and sizes of hyperparameters and other parameters of the model.

\begin{tabular}{|c|c|c|c|c|c|c|}
\hline$\frac{y}{0}$ & $\frac{\bar{D}}{\text { 元 }}$ & 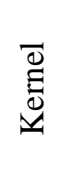 & 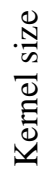 & & 恶 & 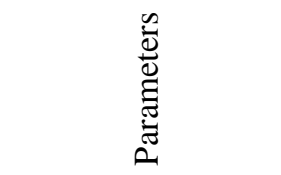 \\
\hline \multirow{6}{*}{1} & 1 & 8 & 3 & same & 2 & $12 \times(8 \times 3+1)=300$ \\
\hline & $2(\mathrm{reLu})$ & & & & & \\
\hline & 3 & 8 & 3 & same & 2 & $12 \times(8 \times 3+1)=300$ \\
\hline & $4(\mathrm{reLu})$ & & & & & \\
\hline & $\begin{array}{c}5(\max \\
\text { pooling) }\end{array}$ & 1 & 2 & same & 1 & $12 \times(1 \times 2+1)=36$ \\
\hline & 6 (dropout) & & & & & 1 \\
\hline \multirow{6}{*}{2} & 7 & 16 & 3 & same & 2 & $12 \times(16 \times 3+1)=588$ \\
\hline & 8 (reLu) & & & & & \\
\hline & 9 & 16 & 3 & same & 2 & $12 \times(16 \times 3+1)=588$ \\
\hline & $10(\mathrm{reLu})$ & & & & & \\
\hline & $\begin{array}{l}11 \text { (max } \\
\text { pooling) }\end{array}$ & 1 & 2 & same & 1 & $12 \times(1 \times 2+1)=36$ \\
\hline & 12 (dropout) & & & & & 1 \\
\hline \multirow{6}{*}{3} & 13 & 32 & 3 & same & 2 & $12 \times(32 \times 3+1)=1164$ \\
\hline & $14(\mathrm{reLu})$ & & & & & \\
\hline & 15 & 32 & 3 & same & 2 & $12 \times(32 \times 3+1)=1164$ \\
\hline & $16(\mathrm{reLu})$ & & & & & \\
\hline & $\begin{array}{l}17 \text { (max } \\
\text { pooling) }\end{array}$ & 1 & 2 & same & 1 & $12 \times(1 \times 2+1)=36$ \\
\hline & 18 (dropout) & & & & & 1 \\
\hline \multirow{6}{*}{4} & 19 & 64 & 3 & same & 2 & $12 \times(64 \times 3+1)=2316$ \\
\hline & 20 (reLu) & & & & & \\
\hline & 21 & 64 & 3 & same & 2 & $12 \times(64 \times 3+1)=2316$ \\
\hline & $22(\mathrm{reLu})$ & & & & & \\
\hline & 24 (dropout) & & & & & 1 \\
\hline & dense & & & & & $27 \times(12 \times 2+1)=675$ \\
\hline otal & & & & & & 9523 \\
\hline
\end{tabular}

Few other classes were cardiac malfunctions where a single pathological pattern occurs in the ECG signal. Taking into account the fact that the signal analysis was limited to 10 seconds only, any other result for these classes could not be expected.

The main limitation of this work was using the first 10 seconds of the signal which was the time covering $83 \%$ of the signals from the available dataset. Such a procedure was determined by the computational time requirements which were cumbersome when using a single PC machine. 
A serious potential improvement has been prepared for this approach and scored well on the validation set, however, it could not be scored on the hidden test set due to the computational time excess. The improvement was based on the many-source CNN approach and consisted of separate networks for: (i) ECG signals themselves, (ii) RR intervals, and (iii) mean beats of the signal. Additionally, the first 2 minutes of each record were used for the analysis.

\section{Conclusions}

We (BioS Team) proposed model for 12-lead ECG model based on convolutional neural networks full test score of $0.279\left(14^{\text {th }}\right.$ place out of 41 in the official ranging of the Challenge). The model may be the first attempt for the further, more complex versions using longer ECG signals as an input and multiple inputs data (such as averaged beat or RR intervals analysis).

Despite obvious limitations, the method used in this work proved versatility of the convolutional neural network approach and its capability and usability for biomedical signal tools and analysis.

\section{Acknowledgments}

We would like to thank our colleagues from the Cardiovascular Physics Group at the Faculty of Physics of Warsaw University of Technology for support.

\section{References}

[1] J. Schläpfer, H. J. Wellens, “Computer-interpreted electrocardiograms: benefits and limitations", J. Am. Coll. Cardiol, vol. 70, no. 9, Aug. 2017, pp. 1183-92.

[2] E. H. Houssein, M. Kilany, A. E. Hassanien, "ECG signals classification: a review", Int. J. Intell. Eng. Inform., vol. 5, no. 4, 2017, pp. 376-96.

[3] T. M. Chen, C. H. Huang, E. S. Shih, Y. F. Hu, M. J. Hwang, "Detection and classification of cardiac arrhythmias by a challenge-best deep learning neural network model", Iscience, vol. 27, no. 3, Mar. 2020, Art. no. 100886.

[4] E. A. Perez Alday, A. Gu, A. Shah, Ch. Robichaux, A.-K. I. Wong, Ch. Liu, F. Liu, A. Bahrami Rad, A. Elola, S. Seyedi, Q. Li, A. Sharma, G. D. Clifford, M. A. Reyna, "Classification of 12-lead ECGs: the PhysioNet/Computing in Cardiology Challenge 2020", Physiol. Meas.

[5] F. Liu, C. Liu, L. Zhao, X. Zhang, X. Wu, X. Xu, Y. Liu, C. Ma, S. Wei, Z. He, J. Li, “An open access database for evaluating the algorithms of electrocardiogram rhythm and morphology abnormality detection", J. Med. Imaging \& Health Infor., vol. 8, no. 7, Sep. 2018, pp. 1368-73.
[6] V. Tihonenko, A. Khaustov, S. Ivanov, A. Rivin, E. Yakushenko, "St Petersburg INCART 12-lead Arrhythmia Database", PhysioBank, PhysioToolkit, and PhysioNet, 2008.

[7] A. Goldberger, L. Amaral, L. Glass, J. Hausdorff, P. C. Ivanov, R. Mark, J. E. Mietus, G. B. Moody, C. K. Peng, H. E. Stanley, "PhysioBank, PhysioToolkit, and PhysioNet: Components of a new research resource for complex physiologic signals", Circulation, vol. 101, no. 23, Jun. 2000, pp. 215-20.

[8] R. Bousseljot, D. Kreiseler, A. Schnabel, "Nutzung der EKG-Signaldatenbank CARDIODAT der PTB über das Internet", Biomed. Eng-Biomed. Te. Vol. 40, no. s1, 1995, pp. 317-8.

[9] P. Wagner, N. Strodthoff, R. D. Bousseljot, D. Kreiseler, F. I. Lunze, W. Samek, T. Schaeffter, "PTB-XL, a large publicly available electrocardiography dataset”, Scientific Data, vol. 7, no. 1, May. 2020, pp. 1-5.

Address for correspondence:

Mateusz Soliński, Koszykowa 75 St. Warsaw 00-662 Poland, mateusz.solinski.dokt@pw.edu.pl 